\title{
MOLTEN STATE OF THERMOPLASTIC RESIN IN AN INJECTION MOULDING BARREL
}

\author{
SO SHIMOKUSUZONO ${ }^{1}$, TATSUYA TANAKA ${ }^{2}$, MASAHIRO SASADA ${ }^{2}$, YUKA HIRAO ${ }^{2}$, \\ KANTA HANAFUSA ${ }^{2} \&$ AKIRA INOUE ${ }^{1}$ \\ ${ }^{1}$ Development Department, TOYO Machinery \& Metal Co. Ltd. of Hyogo, Japan \\ ${ }^{2}$ Applied Materials Engineering Laboratory, Doshisha University of Kyoto, Japan
}

\begin{abstract}
In recent years, polymer products have required superior mechanical properties, a particular strength and dimensional accuracy. One of the most popular moulding methods for polymer materials is injection moulding. Compared to other moulding methods, injection moulding is better at making complicated shapes quicker; however, injection moulding suffers from many kinds of moulding faults. Various solutions to reduce the number of faults on a production line have been developed as a result of many years of studies. However, to further decrease the number of faults, researchers need to focus on the basic state of the plasticized resin. In injection moulding, it is important to determine how uniformly a resin is melted because the uniformity of temperature is related to the characteristics of a product. Therefore, melting completion does not mean that resin is uniform. The uniformity of melting must be accurately determined to achieve high quality. First, this study uncovers some effects of screw behaviours on plasticization. The injection moulding machine performs the metering and injection processes with the screw in the barrel. In the metering process, the screw retreats to the metering stroke while maintaining back pressure. In the injection process, the screw advances to the tip of the nozzle. While the screw repeats these movements, the pellets are provided from the hopper to the moving screw. This method of adding the pellets creates differences in the distance that the resin must travel within the screw channel. The longest distance that the resin must travel during an injection cycle is equal to the metering stroke. The viscosity of the resin depends on the barrel temperature, although the viscosity is also influenced by other moulding conditions. An experiment is performed by changing the metering stroke and the barrel temperature. To understand the condition of the resin just before the injection, an original nozzle with a pressure sensor is produced. Thus, the injection pressure waveform can be recorded during each cycle. As changes in the waveform are reflected in the behaviour of the resin, a non-uniform temperature can be visually confirmed by a disturbance in the waveform.
\end{abstract}

Keywords: injection moulding, molten state, flow analysis.

\section{INTRODUCTION}

Injection moulding is one of the most popular methods for moulding thermoplastic resins. Compared to other moulding methods, injection moulding is better at making a complicated shape in a short takt time; however, injection moulding has many kinds of moulding faults. These faults are caused by the injection moulding machine system. The plasticizing process is not visually captured due to the heating barrel, and the resin, which has viscoelastic properties, is kneaded under high-temperature and high-pressure conditions. Therefore, it has been difficult to accurately obtain the molten state of resin during injection moulding. Reducing the number of faults while considering the inability to view the plasticizing process is very important to improve productivity in injection moulding.

The study and advancement of moulding processing began in the 1500s, and the plasticization phenomenon of resin for extrusion moulding was studied earlier than that for injection moulding. Plasticization phenomena in black-boxed barrels were proposed by Maddock [1] and Street [2]. In this method, the barrel is rapidly cooled during plasticization to solidify the resin, and then the screw is pulled out to analyse the solidified resin in the screw groove. Additionally, based on this experimental result, a plasticization model was 
formulated by Tadmor [3], Tadmor et al. [4] and Tadmor and Klein [5]. In injection moulding, Yokoi et al. [6], [7] carried out a method of attaching a glass window to a barrel or mould to visually determine the behaviour of the resin. This method proved to be a melting model similar to an extruder. Furthermore, the pressure and temperature in both the barrel and the mould were measured by sensors, and a numerical analysis using these experimental data was performed by Guerrier et al. [8] and Atinkaynak et al. [9], [10] to predict resin flow. Many studies have confirmed whether the resin is melted in a barrel and determined the flow behaviour of resin in a metal mould. As a result of these studies, some techniques for reducing the number of moulding faults have been established. However, no complete solution has yet been reached. Few specific research studies have evaluated the state of the molten resin in injection moulding. This evaluation is necessary to produce high-quality mouldings because the molten state at the completion of plasticization is greatly related to the production quality. Researchers should consider whether the molten state is uniform as well as whether the resin is molten before it is moulded. Chen et al. [11] reported how the viscosity of the molten resin in injection moulding affects the moulded article. Therefore, this study establishes a method to evaluate whether the parameters of temperature, density and viscosity of a resin are uniform immediately before being injected into a metal mould. This method is then compared to the energy obtained by a flow analysis.

The injection moulding machine performs the metering and injection processes by using the screw in the barrel. During the metering process, the screw retreats to the metering stroke while maintaining back pressure. During the injection process, the screw advances to the tip of the nozzle. While the screw repeats these movements, the pellets are provided from the hopper to the moving screw. This method of adding the pellets creates differences in the distance that the resin must travel within the screw channel. The longest distance that the resin must travel during an injection cycle is equal to the metering stroke. This study was conducted to verify the influence that the difference in screw length, which is caused by the retraction behaviour of the screw, has on the resin.

\section{EXPERIMENT}

\subsection{Flow simulation [12]}

It is thought that the amount of energy that the resin receives from shear stress during a plasticization process influences the molten state of the resin in injection moulding. In this study, to investigate the molten state of plastic resin, a flow simulation was performed using POLYFLOW (ANSYS, Inc.).

\subsubsection{Simulation conditions}

The shear stress and flow path of the molten resin can be calculated by making the following assumptions.

1. The molten resin is an incompressible, non-isothermal, and non-Newtonian fluid.

2. Gravitational forces are ignored during the simulation because the molten resin is a highly viscous fluid.

3. The fluid completely melts, and the flow channel is completely filled.

4. There is no slip on the surface of the solid.

The viscosity of the polymer around the screws was modelled by the Bird-Carreau law (eqn (1) below), and the properties of polypropylene at $180^{\circ} \mathrm{C}, 200^{\circ} \mathrm{C}$ and $220^{\circ} \mathrm{C}$ were applied 


$$
\mathrm{F}(\dot{\gamma})=\eta_{\infty}+\left(\eta_{0}-\eta_{\infty}\right)\left(1+\lambda^{2} \dot{\gamma}^{2}\right)^{\frac{n-1}{2}},
$$

where $\eta_{0}$ is the zero-shear-rate viscosity, $\lambda$ is the time constant, $\dot{\gamma}$ is the shear rate and $n$ is the power law index. The shear viscosity and the shear velocity were measured using a capillary rheometer.

The volumetric flow rate $Q$ was calculated using the following expression:

$$
\mathrm{Q}=\frac{\pi r^{2} h}{t}
$$

where $t$ is the charge time, $r$ is the screw radius and $h$ is the injection stroke. Table 1 shows the volumetric flow rate $Q$ in the flow channel for each temperature condition.

Table 1: Volumetric flow rate in the flow channel of the storage temperature condition.

\begin{tabular}{|c|c|}
\hline Temperature $\left({ }^{\circ} \mathrm{C}\right)$ & Volumetric flow rate $\left(\mathrm{mm}^{3} / \mathrm{sec}\right)$ \\
\hline 180 & 2083 \\
\hline 200 & 2121 \\
\hline 220 & 2106 \\
\hline
\end{tabular}

The plasticization process in each screw design was evaluated by a particle tracking method. The residence time or plasticization time could be used for this purpose. The particle tracking was conducted using a Lagrangian method with a second-order accuracy in a space consisting of two steps

$$
\begin{gathered}
\text { 1st step: } \quad x_{i}^{1 / 2}=x_{i}^{0}+\frac{1}{2} u_{i}^{0} \Delta t, \\
\text { 2nd step: } \quad x_{i}^{1}=x_{i}^{1 / 2}+\Delta t\left(u_{i}^{1 / 2}-\frac{1}{2} u_{i}^{0}\right),
\end{gathered}
$$

where $x_{i}$ is the position of the particle, $u_{i}$ is the velocity vector and $\Delta t$ is the time increment.

To determine the residence time distribution of the particles, 1000 particles were charged via the inflow port of the hopper for $100 \mathrm{~s}$. The trajectory of each particle throughout the screw was calculated, and the histories of the individual particle coordinates, the stress and the strain rates were recorded by particle tracking. The amount of energy that the resin receives from shear stress during plasticization was calculated using eqn (5), and this value was used for one index to evaluate the uniformity of the molten resin

$$
U=\int_{0}^{t_{p}} \tau \cdot \dot{\gamma} \mathrm{dt}
$$

where $\tau$ is the shear stress and $t_{p}$ is the particle residence time.

The initial temperature of the fluid was assumed to be $180^{\circ} \mathrm{C}, 200^{\circ} \mathrm{C}$ or $220^{\circ} \mathrm{C}$. The screw rotation speed was $100 \mathrm{rpm}$. The screw during a plasticization process retreats smoothly. However, in this study, the analysed retreat distances of the screw were $0 \mathrm{~mm}, 24 \mathrm{~mm}$, $48 \mathrm{~mm}$, and $72 \mathrm{~mm}$, and the state was changed by the length of the screw; the results were obtained as a fragmentary state of 1 cycle.

\subsection{Evaluation of the molten resin state}

\subsubsection{Materials and equipment}

For this investigation, polypropylene (grade J108M) was selected. The employed injection moulding machine was a 40-ton clamping force model (TOYO PLASTAR ET-40V) with an 
all-electric drive and a screw diameter of $24 \mathrm{~mm}$. A spiral-shaped metal mould was used to avoid needing to be changed for some metering strokes. The moulding conditions used for the fabrication of the test pieces are shown in Table 2. These conditions are standard for the applied mould. Ten shots were used for the experimental evaluation. This experiment was performed by changing the metering stroke and the barrel temperature.

Table 2: Moulding condition.

\begin{tabular}{|c|c|c|c|}
\hline Screw rotation speed (rpm) & \multicolumn{3}{|c|}{100} \\
\hline Injection speed $(\mathrm{mm} / \mathrm{s})$ & \multicolumn{3}{|c|}{5} \\
\hline Back pressure $(\mathrm{MPa})$ & \multicolumn{3}{|c|}{5} \\
\hline Metering stroke $(\mathrm{mm})$ & 24 & 48 & 72 \\
\hline Barrel temperature $\left({ }^{\circ} \mathrm{C}\right)$ & 180 & 200 & 220 \\
\hline Tool temperature $\left({ }^{\circ} \mathrm{C}\right)$ & \multicolumn{3}{|c}{40} \\
\hline Cooling time $(\mathrm{sec})$ & \multicolumn{3}{|c}{25} \\
\hline
\end{tabular}

\subsubsection{Evaluation method}

To judge the molten state of the resin, the maximum injection pressure was recorded. This pressure is the value of the resistance that the screw receives at the moment of injection. Additionally, the flowing distance of the resin was determined by putting the spiral product on a protractor and adding up the number of turns and the remaining angle.

\section{RESULTS AND DISCUSSION}

\subsection{Flow simulation}

\subsubsection{Simulation of the full-length screw}

In the case of $200^{\circ} \mathrm{C}$, the energy that each particle received between the inflow and outflow was $6.05 \mathrm{MJ} / \mathrm{m}^{3}$. Fig. 1 shows the energy that the particles received in 3 different zones: the feed zone, compression zone and metering zone. This figure shows that the energy that the particles receive from the screw is highest in the feed zone. If the shear rate becomes large, the energy that the particles receive also becomes large, as shown in eqn (5). The shear rate is a geometric parameter that is influenced by the screw shape and screw rotation speed. The shear rate increases when the ditch of the screw is shallow. Therefore, the shear rate usually becomes highest in the metering zone. However, the energy in the feed zone is the highest. The feed zone accounts for approximately half of the whole screw, and the residence time in the feed zone is longer than that in the two other zones. For the analysis, the resin is supposed to completely melt at the inflow. In actuality, after a solid resin pellet is placed in the hopper, the pellet is transported forward by the frictional force of the screw and barrel while receiving heat from the hot barrel. After fusion begins, the energy the resin receives changes to viscous shear stress. The temperature of the resin is low near the hopper, and the resin viscosity is in a high state. The shear stress that is added to the materials becomes large when the resin viscosity is high. As a result, the energy that the resin receives substantially increases. From this phenomenon, the ratio of mechanical energy that the resin receives in the feed zone is very large. In other words, it is thought that the decrease in the length of the screw in the feed zone from the retreating behaviour has a large influence on the plasticization and melting of the resin. 


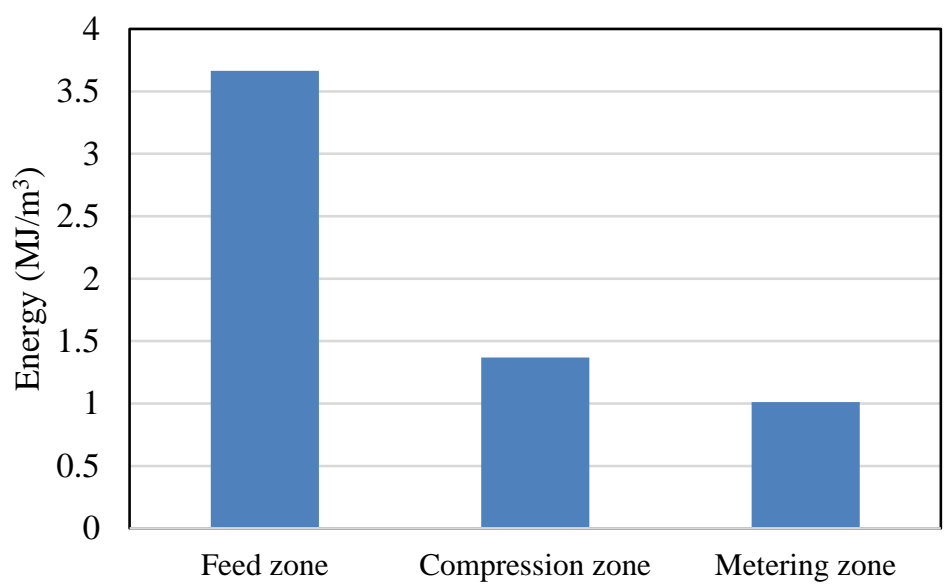

Figure 1: Increase in energy within each zone.

\subsubsection{Simulation of the screw in each retreat distance condition}

An analysis was performed that varied the length of the screw according to the distance that the screw retreated. A result provided by particle tracking is shown in Fig. 2. The horizontal axis is the value of mechanical energy that each particle received, and the vertical axis is the number of particles in the energy zone. The results confirmed that the particles received less energy when the retreat distance of the screw was large. Therefore, the distribution of the energy was shown to become wider when the retreat distance of the screw was larger. From the actual moulding, it was expected that an irregularity occurred in the uniformity of the molten resin. The mean value of the energy for each screw length is shown in Fig. 3. From this figure, it was confirmed again that the energy decreased when the retreat distance of the screw increased.

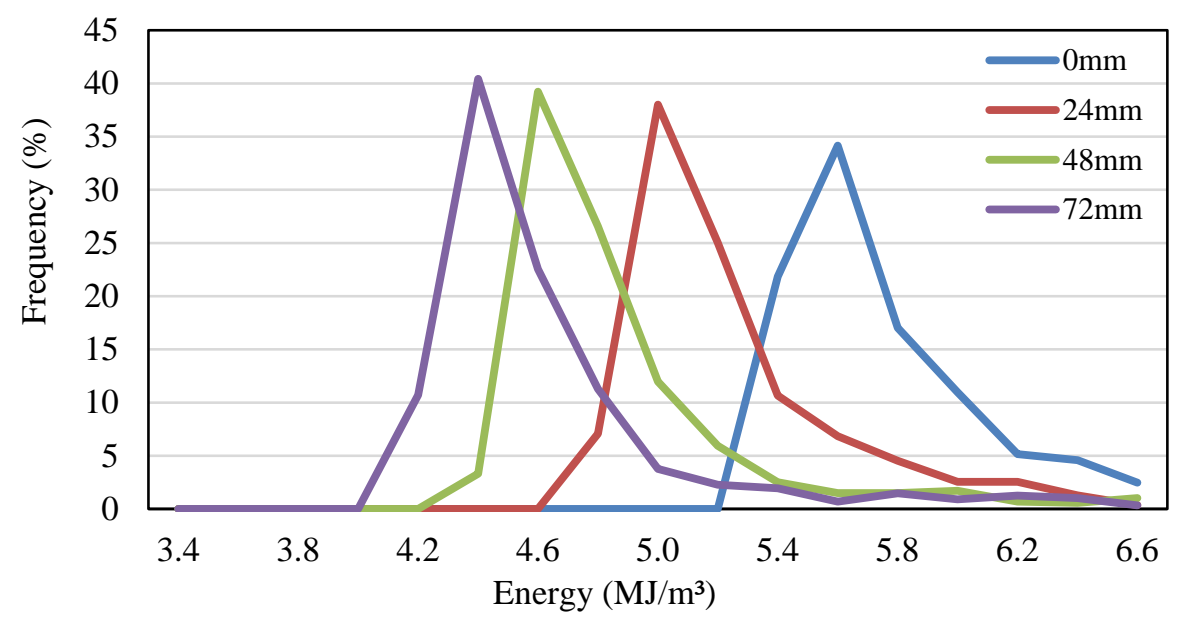

Figure 2: Histogram of each retreat distance condition. 


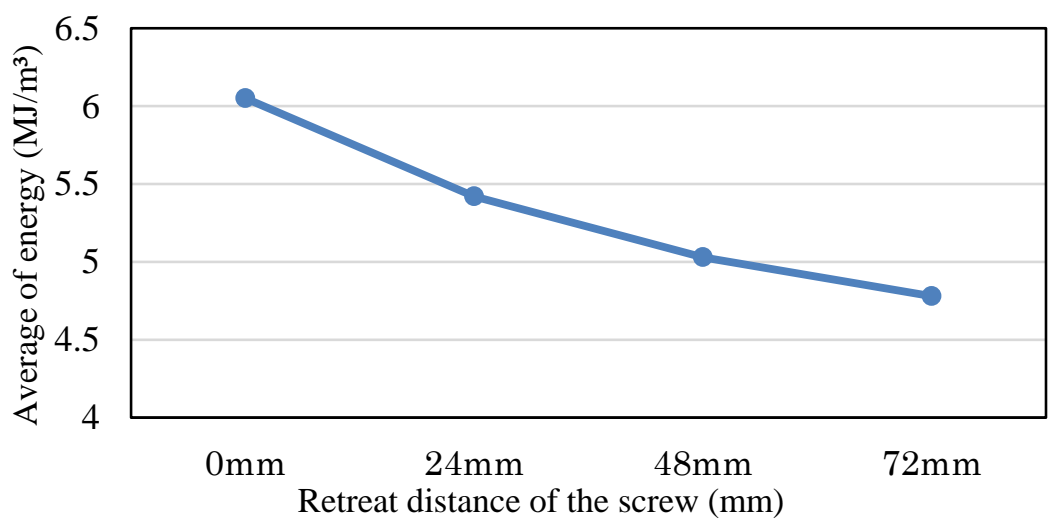

Figure 3: Average energy value at each retreat distance condition.

\subsubsection{Simulation of each temperature condition}

The difference in the amount of energy when the temperature was changed is shown in Fig. 4. The retreat distance of the screw is shown on the horizontal axis, and the decrease in the amount of energy is shown on the vertical axis. The results confirmed that the difference in the energy increased when the temperature was lower, and it was confirmed that the difference in the energy increased when the retreat distance was larger. This phenomenon occurs because the mechanical energy that the resin receives increases as a result of the shear stress, and the resin with lower temperature has a larger effect from the shear stress. Additionally, as the retreat distance of the screw increased, there was a larger change in the energy.

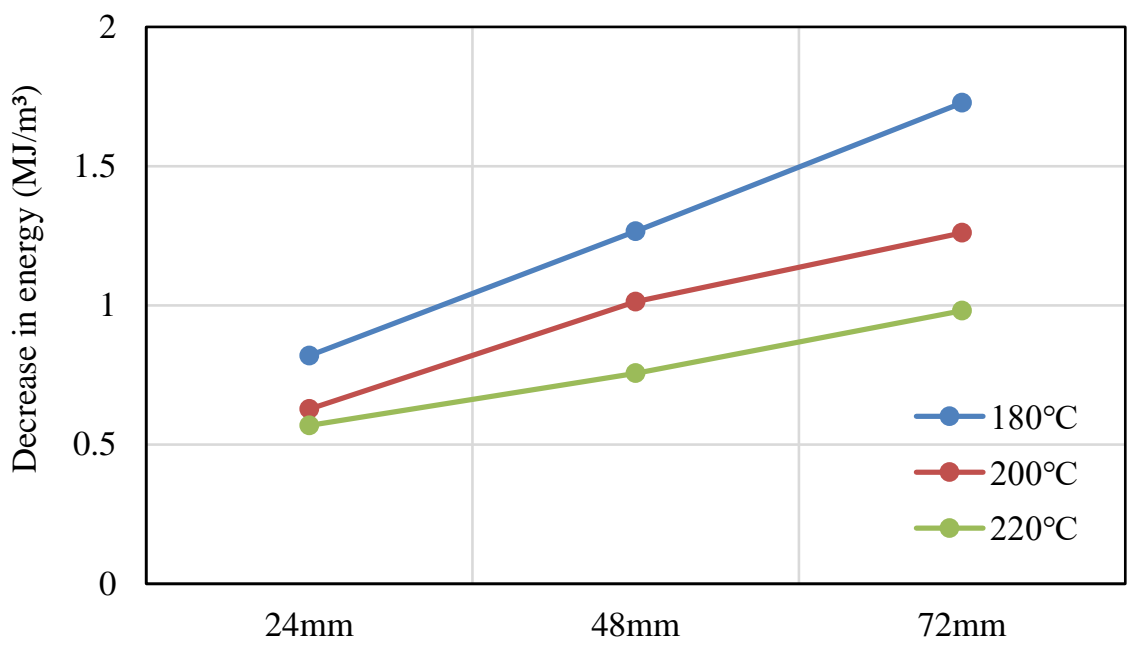

Retreat distance of the screw (mm)

Figure 4: Energy difference from the full-length screw at each retreat distance. 


\subsection{Evaluation of the molten resin state}

The average maximum injection pressure is shown in Fig. 5. The relationship between the barrel temperature, metering stroke and maximum injection pressure was evaluated. According to the results, the maximum injection pressure decreased as the barrel temperature increased, and the maximum injection pressure increased as the metering stroke increased. The cause of the former is that the viscosity decreases as the barrel temperature increases. For the cause of the latter, the influence of the mould on the flow resistance increases due to the increase in the metering stroke. Therefore, the standard deviation is calculated as the degree of variation per shot.

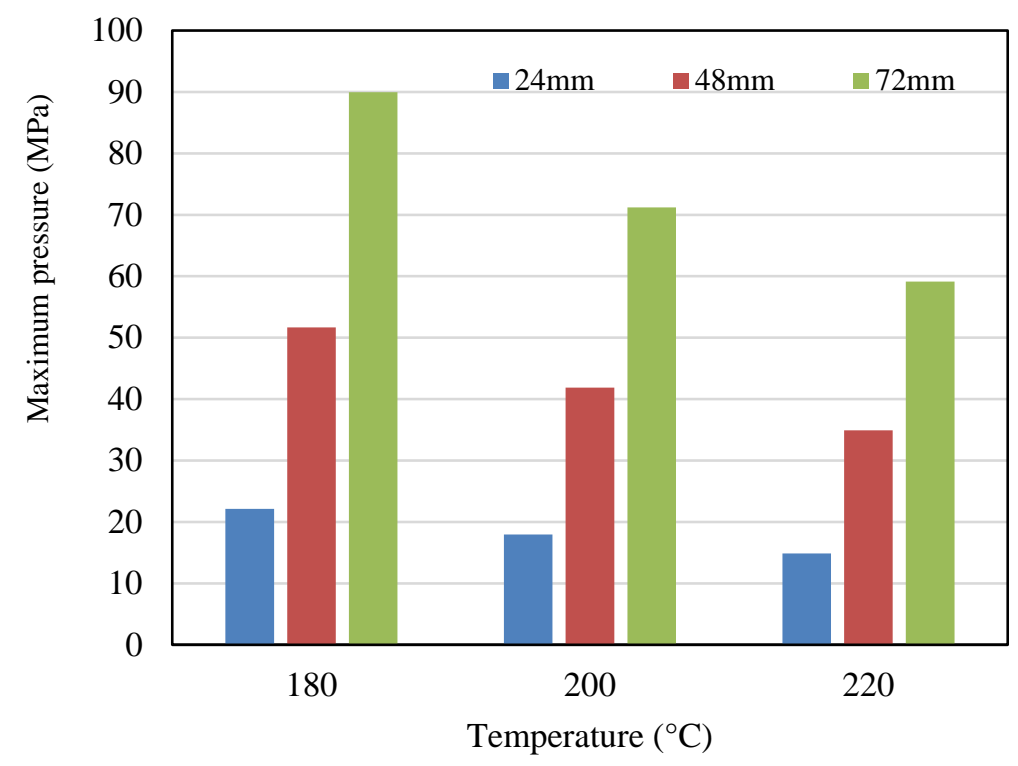

Figure 5: Average maximum injection pressure with respect to the barrel temperature and metering stroke.

The standard deviation of the maximum injection pressure for each barrel temperature is shown in Fig. 6(a), (b) and (c). At the barrel temperature of $180^{\circ} \mathrm{C}$, the standard deviation of the maximum injection pressure increases as the metering stroke increases. However, at $220^{\circ} \mathrm{C}$, the state of the resin is considered stable because the standard deviations were approximately the same for all metering stroke values. This tendency was similarly seen in the standard deviation of the flowing distance of the moulded particle. In other words, the molten state of the resin is affected by the metering stroke as the barrel temperature decreases. Before we conclude that the standard deviation becomes larger when the metering stroke increases, it is necessary to consider the residence time. This consideration of residence time is important because when the metering stroke is increased, the amount of plasticization increases, and the time required for the resin to be ejected from the hopper and injected is shortened. Therefore, we need to find a way to analyse the standard deviation that is not influenced by the residence time. 


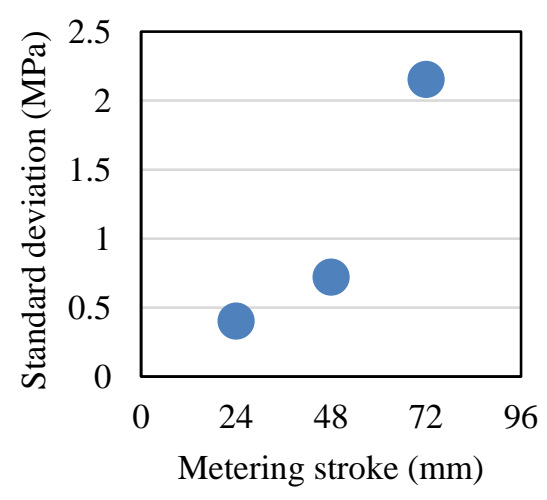

(a)

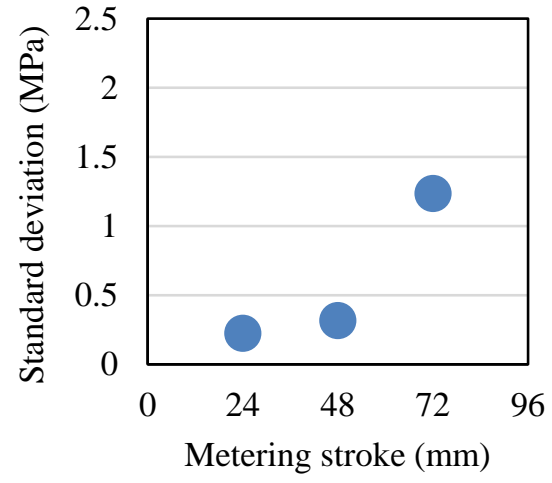

(b)

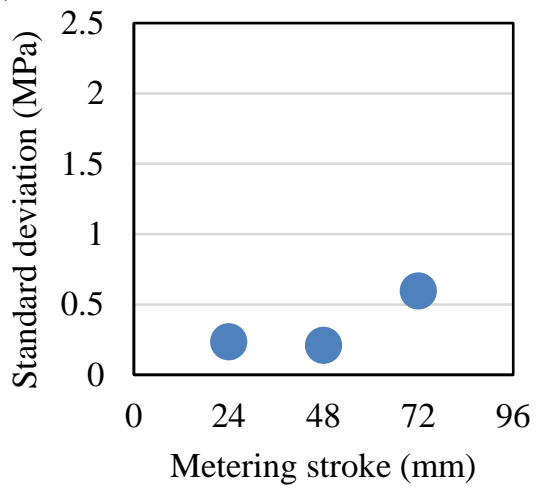

(c)

Figure 6: Deviation of the maximum injection moulding pressure for each barrel temperature. (a) $180^{\circ} \mathrm{C}$; (b) $200^{\circ} \mathrm{C}$; and (c) $220^{\circ} \mathrm{C}$.

It may be difficult to compare the molten state of the resin because the injection pressure is influenced by the shape of the metal mould. Therefore, we tried an experiment wherein the resin was injected into the air, similar to a purge, instead of injecting into a mould. A purge is an operation for replacing the resin in the barrel when changing materials or removing the remaining resin in the barrel. However, the back pressure is very important because it largely relates to the molten state of the resin. In this experiment, the measuring process was performed by touching the nozzle and applying back pressure before injecting. Additionally, a pressure sensor (NP465XL-1/2-20MPa of DYNISCO JAPAN) was installed at the tip of the nozzle to determine the state of the resin from a closer position. The pressure was measured every $100 \mathrm{~ms}$. We obtained a waveform by connecting the pressure points. The pressure waveform obtained in one cycle is shown in Fig. 7. There are two places where the pressure increases. The first pressure increase occurs at the time of measuring, which exhibits the same pressure as the back pressure. The second pressure increase occurs at the time of injection. This pressure waveform was extracted for each condition and evaluated. The pressure waveforms at the injection section are shown in Fig. 8(a), (b) and (c). The resin flow immediately after the start of injection was the same regardless of the metering stroke; the pressure waveforms at $24 \mathrm{~mm}$ and $72 \mathrm{~mm}$ were similarly shaped, but the pressure waveform at $24 \mathrm{~mm}$ was half as long as the waveform at $72 \mathrm{~mm}$. Therefore, the waveform just before 
the end of injection is the focus of evaluating the difference in the molten state of the resin by the metering stroke. However, in this experiment, the nonuniformity of the resin did not appear in the disturbance of the waveform; as the nozzle in this study is generally used in injection moulding, the pressure may not be sufficient to indicate resin nonuniformity.

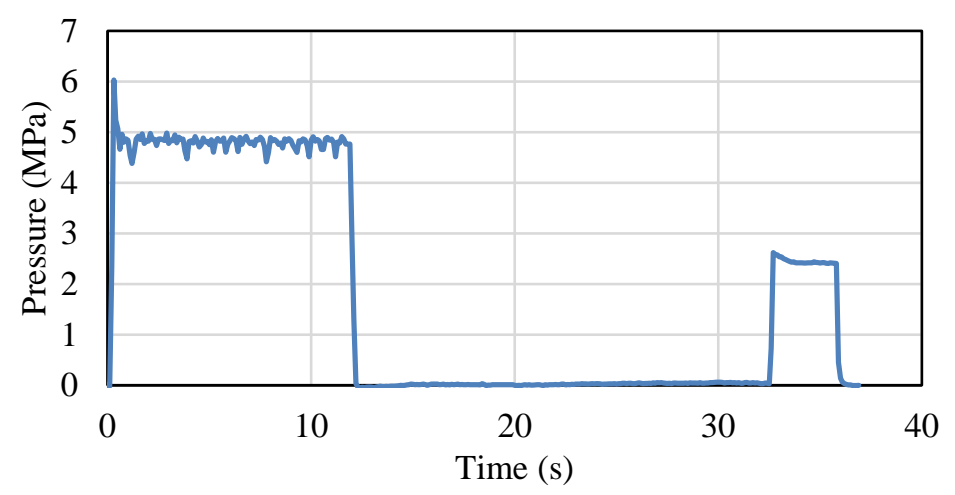

Figure 7: Sample of the pressure waveform in a cycle.

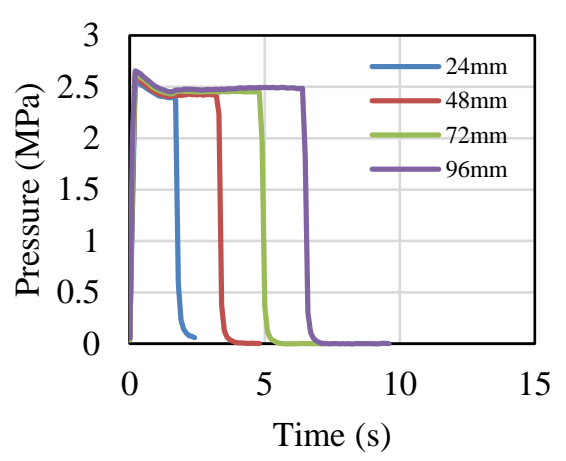

(a)

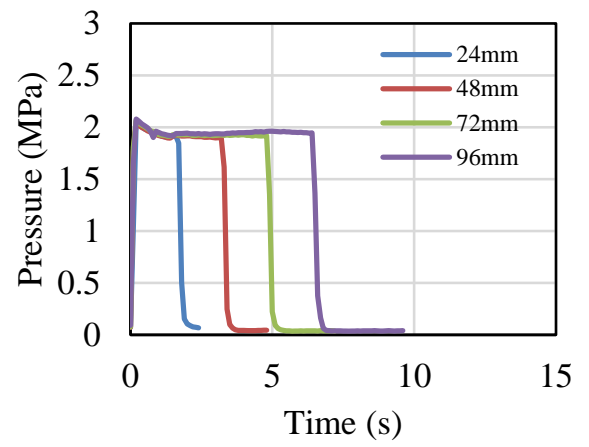

(b)

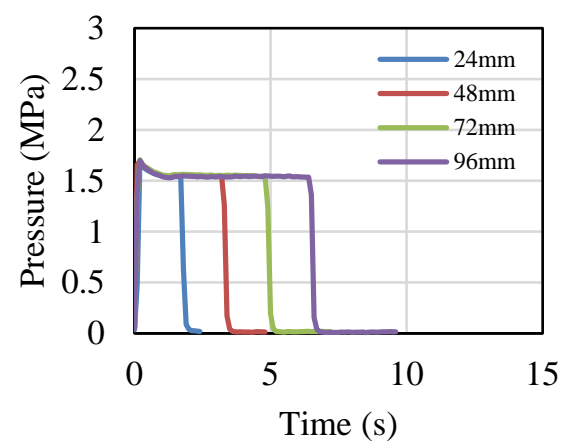

(c)

Figure 8: Pressure waveform in the injection section. (a) $180^{\circ} \mathrm{C}$; (b) $200^{\circ} \mathrm{C}$; and (c) $220^{\circ} \mathrm{C}$. 
Therefore, we investigated the unevenness of the data under each condition. Fig. 9 shows a result provided under conditions of $72 \mathrm{~mm}$ at each temperature. As a result, it was confirmed that the unevenness of the pressure decreased because the temperature of the barrel rose. This observation was well consistent with a result provided by analysis.

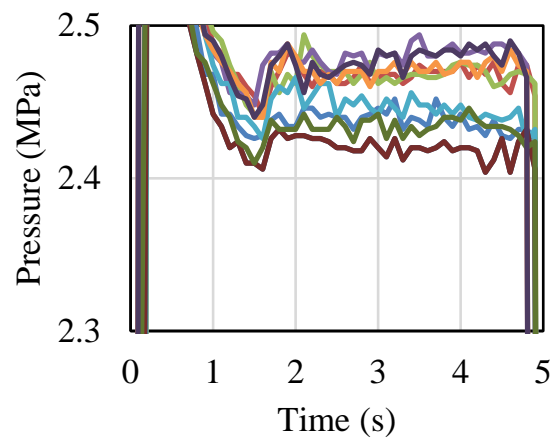

(a)

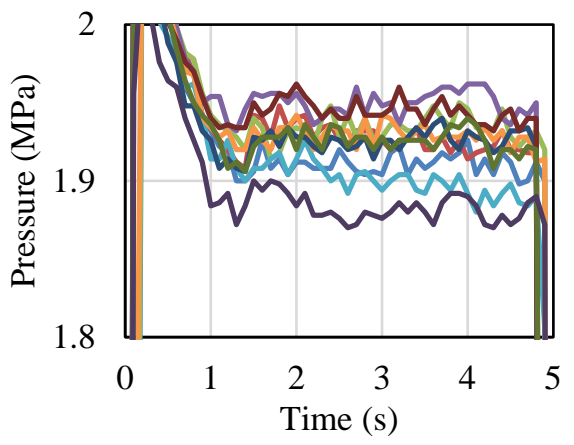

(b)

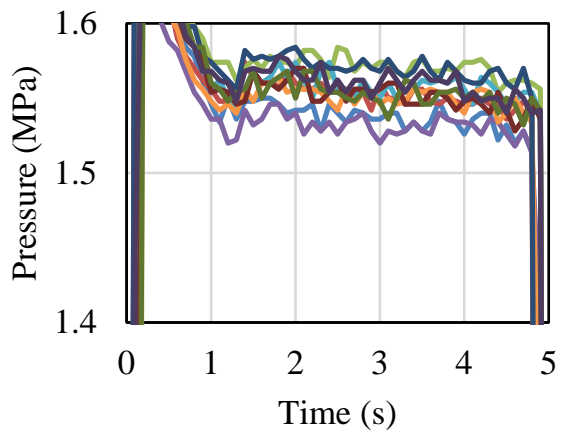

(c)

Figure 9: Results provided under conditions of $72 \mathrm{~mm}$. (a) $180^{\circ} \mathrm{C}$; (b) $200^{\circ} \mathrm{C}$; (c) $220^{\circ} \mathrm{C}$.

\section{CONCLUSIONS}

In this paper, we conducted experiments to verify how the difference in screw length (i.e., where the resin passes by the retracting behaviour of the screw) affects the molten state. This experimentation aims to establish a method that can evaluate whether the molten state of the resin is uniform just before being injected into the metal mould.

A flow analysis was performed, and the results showed that the difference in the energy that the resin received grew large when the retreat distance of the screw was large. It was thought that this difference in energy had an influence on the uniformity of the resin. Furthermore, it was confirmed that the influence was more substantial when the temperature was lower.

Since the data trend of the flowing distance of the moulded product coincides with that obtained from injection moulding, it can be said that it is effective to evaluate the pressure at the time of injection to stabilize the molten state of the resin. Although there was a phenomenon in which the standard deviation of the maximum injection pressure became larger as the metering stroke increased, at the present stage, it cannot be concluded that the increased standard deviation was caused by the variations in the metering stroke. It was found 
that the residence time must be considered in future experiments. We tried an experiment to inject resin into the air, similar to a purge, instead of injecting the resin into a mould. As a result, the state of the resin was not confirmed even if we compared the experimental means. However, we were able to grasp the unevenness of the resin in a molten state when we investigated the state of the pressure under every condition in detail. This finding was consistent with an analytical result.

\section{REFERENCES}

[1] Maddock, B.H., A Visual Analysis of Flow and Mixing in Extruder Screws. SPE Journal, 15, 1959.

[2] Street, L.F., Plasticating extrusion. International Plastics Engineering, 1, 1961.

[3] Tadmor, Z., Fundamentals of plasticating extrusion. I. A theoretical model for melting. Polymer Engineering and Science, 6(3), pp. 185-190, 1966.

[4] Tadmor, Z., Duvdevani, H.J. \& Klein, I., Melting in plasticating extruders theory and experiments. Polymer Engineering and Science, 7(3), pp. 198-217, 1967.

[5] Tadmor, Z. \& Klein, I., Engineering Principles of Plasticating Extrusion, Van Nostrand Reinhold Co., 1970.

[6] Yokoi, H., Takatsugi, S. \& Shiraishi, W., Development of a small-sized glass-inserted heating cylinder for visualization and temperature distribution measurement in injection molding. Institute of Industrial Science, 52(9), pp. 410-413, 2000.

[7] Yokoi, H., Hayasaki, S., Kuroda, A. \& Tatsuno, M., Visualization of screwplasticating process of plastics pellets by glass-inserted heating cylinder. Journal of Flow Visualization and Image Processing, 7, pp. 231-241, 2000.

[8] Guerrier, P., Tisello, G. \& Hattel, J.H., Flow visualization and simulation of the filling process during injection molding. CIRP Journal of Manufacturing Science and Technology, 16, pp. 12-20, 2017.

[9] Altinkaynak, A., Gupta, M., Spalding, M.A. \& Crabtree, S.L., Melting in a single screw extruder: experiments and 3d finite element simulations. International Polymer Processing, 26(2), pp. 182-196, 2011.

[10] Altinkaynak, A., Gupta, M., Spalding M.A. \& Crabtree, S.L., An investigation of melting and metering sections in a single-screw extruder. SPE ANTEC The. Papers, 60, pp. 1135-1141, 2014.

[11] Chen, J.Y., Yang, K.J. \& Huang, M.S., Online quality monitoring of molten resin in injection molding. International Journal of Heat and Mass Transfer, 122, pp. 681693, 2018.

[12] Inoue, A. Morita, K., Tanaka, T., Arao Y. \& Sawada, Y., Effect of screw design on fiber breakage and dispersion in injection-molded long-glass-fiber-reinforced polypropylene. Journal of Composite Materials, 49(1), pp. 75-84, 2015. 